\title{
GROSS-KOHNEN-ZAGIER THEOREM FOR HIGHER WEIGHT FORMS
}

\author{
Hui Xue
}

\begin{abstract}
We calculate the height pairing between higher weight Heegner cycles associated to distinct imaginary quadratic fields. Under the positive definiteness hypothesis of the height pairing we prove a Gross-Kohnen-Zagier theorem for higher weight modular forms.
\end{abstract}

\section{Introduction}

Let $X_{0}(N)$ be the usual modular curve over $\mathbb{Z}$. Let $\mathcal{O}_{D}$ be the order of discriminant $D<0$ in an imaginary quadratic field $K_{D}=\mathbb{Q}(\sqrt{D}) \subset \mathbb{C}$. Assume that $(N, D)=1$ and all prime factors of $N$ split in $K_{D}$. The theory of complex multiplication defines certain points $x \in X_{0}(N)\left(H_{D}\right)$. These special points are called Heegner points of discriminant $D$, and $H_{D}$ is the ring class field corresponding to the order $\mathcal{O}_{D}$, see [7] for details.

Following the notation of [8], such a Heegner point $x$ is determined by the residue class of $r$ modulo $2 N$, where $r$ satisfies

$$
r^{2} \equiv D(\bmod 4 N)
$$

More precisely, let $\tau$ be the root in the upper half plane $\mathbb{H}$ of the equations

$$
a \tau^{2}+b \tau+c=0, \quad a>0, \quad N \mid a, \quad b^{2}-4 a c=D, \quad \text { and } b \equiv r(\bmod 2 N),
$$

then the image of $\tau$ in $X_{0}(N)(\mathbb{C})$ is a Heegner point defined over $H_{D}$. There are exactly $h_{D}=\left[H_{D}: K_{D}\right]$ such images, permuted transitively by $\operatorname{Gal}\left(H_{D} / K_{D}\right)$, and their formal sum is denoted by $P_{D, r}$. The Zariski closure of $P_{D, r}$ in $X_{0}(N)_{\mathbb{Z}}$ is denoted by $\underline{P}_{D, r}$.

Each Heegner point in $P_{D, r}$ can also be interpreted as an isogeny of elliptic curves $\left(E \stackrel{\phi}{\rightarrow} E^{\prime}\right)$ both of which have $\mathrm{CM}$ by the same order $\mathcal{O}_{D}$, and the kernel of $\phi$ is annihilated by the primitive ideal $\left(N, \frac{r+\sqrt{D}}{2}\right)$ of norm $N$ in $\mathcal{O}_{D}$.

Let

$$
S_{2 k}^{-}\left(\Gamma_{0}(N)\right)=\left\{g \in S_{2 k}\left(\Gamma_{0}(N)\right) \mid\left(-N z^{2}\right)^{-k} g\left(\frac{-1}{N z}\right)=-g(z)\right\}
$$

be the subspace of cuspforms of weight $2 k$ which have eigenvalue $(-1)$ under the Fricke involution. If $f \in S_{2 k}^{-}\left(\Gamma_{0}(N)\right)$ is a normalized newform, then $L(f, s)$ has minus sign in its functional equation under $s \mapsto 2 k-s$. By the result of [10] there is a nonzero Jacobi form $\phi_{f} \in J_{k+1, N}^{\text {cusp }}$, unique up to a scalar multiple, which has the same eigenvalues as $f$ for Hecke operators $T_{m}$ with $(m, N)=1$. The Jacobi form $\phi_{f}$ can be

Received by the editors March 30, 2010.

2000 Mathematics Subject Classification. 11F67, 11G50. 
chosen to have Fourier coefficients in the same totally real coefficient field of $f$. We write the Fourier expansion of $\phi_{f}$ as

$$
\phi_{f}(\tau, z)=\sum_{\substack{n, r \in \mathbb{Z} \\ r^{2} \leq 4 n N}} c(n, r) q^{n} \zeta^{r}
$$

where $\tau \in \mathbb{H}, z \in \mathbb{C}, q=e^{2 \pi i \tau}$ and $\zeta=e^{2 \pi i z}$.

When $k=1$, a well-known result of Gross-Kohnen-Zagier [8] shows that for each fundamental discriminant $D,\left(y_{D, r}^{*}\right)_{f}$ is essentially equal to $c(n, r)$ times a fixed $y_{f}$ in the space $J_{0}(N)(\mathbb{Q}) \otimes \mathbb{R}$, where $\left(y_{D, r}^{*}\right)_{f}$ is the $f$-isotypical component of the divisor class

$$
y_{D, r}^{*}=\left(P_{D, r}-h_{D} \infty\right)+\overline{\left(P_{D, r}-h_{D} \infty\right)},
$$

and $n=\frac{r^{2}-D}{4 N}$. Our main goal is to generalize this result to $f$ of general weight $2 k$.

For this purpose we first review the construction of Kuga-Sato varieties and Heegner cycles on them associated to weight $2 k$ modular forms. After that we calculate the height pairing between the Heegner cycles and compare them with the Fourier coefficients of a modular form $F$, whose inner product with $f$ gives (essentially) the central derivative $L^{\prime}(f, k)$. In the end, if we assume further that the height pairing between Heegner cycles is positive definite, we are able to prove a higher weight analogue of the Gross-Kohnen-Zagier theorem. It would be interesting to find an unconditional proof, for instance, by generalizing the approach taken in Borcherds [3].

\section{Kuga-Sato varieties and CM cycles}

Let $N^{\prime}$ with $N \mid N^{\prime}$ be an auxiliary number which is a product of two coprime integers greater than 2. There is a natural morphism $\pi: \mathcal{E}\left(N^{\prime}\right) \rightarrow X\left(N^{\prime}\right)$ of regular, flat and projective schemes, such that $\pi$ makes $\mathcal{E}\left(N^{\prime}\right)$ a universal semistable elliptic curves with full level $N^{\prime}$ structures over $X\left(N^{\prime}\right)$. The universal elliptic curve with level $N^{\prime}$ structures is denoted by $\mathcal{E}_{0}\left(N^{\prime}\right)$ and the corresponding moduli scheme is denoted by $Y\left(N^{\prime}\right)$.

The $(2 k-2)$-tuple fiber product $\mathcal{E}\left(N^{\prime}\right)^{2 k-2}$ over $X\left(N^{\prime}\right)$ has a canonical resolution, denoted by $Y_{k}\left(N^{\prime}\right)$, which is described in Zhang [11] (similar to that in Deligne [4] over Q). The scheme $Y_{k}\left(N^{\prime}\right)$ is the Kuga-Sato variety that will be used for the construction of CM cycles or Heegner cycles in this paper.

The Hecke correspondences $T_{m}$ for $\left(m, N^{\prime}\right)=1$ are defined as follows. Let $Y\left(N^{\prime}, m\right)$ denote the moduli scheme classifying elliptic curves $E$ with level $N$ structure and a cyclic isogeny $E \rightarrow E^{\prime}$ of degree $m$. Let $\mathcal{E}_{0}\left(N^{\prime}, m\right)$ be the universal elliptic curve over $Y\left(N^{\prime}, m\right)$ and $\mathcal{E}_{0}\left(N^{\prime}, m\right) \stackrel{\psi}{\rightarrow} \mathcal{E}_{0}^{\prime}\left(N^{\prime}, m\right)$ be the universal cyclic $m$-isogeny. We have the following diagram

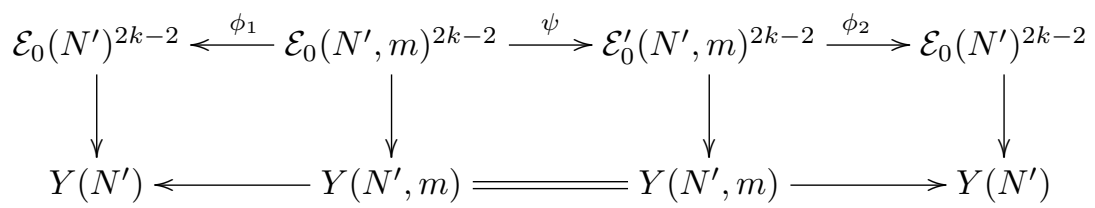


The Hecke correspondence $T_{m}$ is defined to be the Zariski closure in $\mathcal{E}\left(N^{\prime}\right)^{2 k-2} \times$ $\mathcal{E}\left(N^{\prime}\right)^{2 k-2}$ (or in $Y_{k}\left(N^{\prime}\right)$ to be more precise) of the correspondence $\phi_{1 *} \psi^{*} \phi_{2}^{*}$ on $\mathcal{E}_{0}\left(N^{\prime}\right)^{2 k-2}$.

Next we want to define the notion of CM Chow cycles of weight $2 k$, full level $N^{\prime}$ and discriminant $D$. For this purpose let $R$ be an integral domain which is flat over $\mathbb{Z}$ and is unramified over all primes dividing $N^{\prime}$. In later applications we always take $R$ to be $\mathcal{O}_{H_{D}}$ or its local completion with $\left(D, N^{\prime}\right)=1$. By the choice of $R$ we know the base change $Y_{k}\left(\Gamma\left(N^{\prime}\right)\right)_{R}$ is again regular. Denote the generic point of Spec $R$ by $\eta$. Let $\underline{x} \in X\left(N^{\prime}\right)(R)$ be such that the corresponding elliptic curve $E$ over Spec $R$ has $\mathrm{CM}$ by $\mathcal{O}_{D}$. Fix an embedding $\tau=\tau_{E}: \operatorname{End}_{R}(E) \rightarrow \mathbb{C}$. Let $\sqrt{D}$ be the element in $\operatorname{End}_{R}(E)$ with $\tau_{E}(\sqrt{D})=\sqrt{D}$ in $\mathcal{O}_{D}$. Let $\Gamma_{D}$ be the graph of the multiplication by $\sqrt{D}$ and write

$$
Z(E)=\Gamma_{D}-(E \times\{0\})-|D|(\{0\} \times E) .
$$

The $(k-1)$-tuple product $Z(E)^{k-1}$ defines a cycle of codimension $k-1$ in $E^{2 k-2}$. Define

$$
W_{k}(\underline{x}):=W_{k}(E)=\sum_{\sigma \in G_{2 k-2}} \operatorname{sgn}(\sigma) \sigma^{*}\left(Z(E)^{k-1}\right)
$$

and let

$$
S_{k}(\underline{x}):=S_{k}(E)=c \cdot W_{k}(E)
$$

where the positive constant $c$ is such that the self-intersection of $S_{k}\left(E_{\eta}\right)$ in $E_{\eta}^{2 k-2}$ is $(-1)^{k-1}$. After base changing to $R$ we have the following natural morphisms

$$
Y_{k}\left(N^{\prime}\right)_{R} \rightarrow \mathcal{E}\left(N^{\prime}\right)_{R}^{2 k-2} \rightarrow X\left(N^{\prime}\right)_{R}
$$

Through the natural morphism $E^{2 k-2} \rightarrow \mathcal{E}\left(N^{\prime}\right)_{R}^{2 k-2}$, the cycle $S_{k}(\underline{x})$ can be viewed as living in $\mathcal{E}\left(N^{\prime}\right)_{R}^{2 k-2}$, and as living in $Y_{k}\left(N^{\prime}\right)_{R}$ as well (because CM elliptic curves have potentially good reductions). The codimension of $S_{k}(\underline{x})$ in $Y_{k}\left(N^{\prime}\right)_{R}$ becomes $k$. The class of $S_{k}(\underline{x})$ in $C h^{k}\left(Y_{k}\left(N^{\prime}\right)_{R}\right)$ is denoted by $s_{k}(\underline{x})$, and is called a CM Chow cycle of weight $2 k$, full level $N^{\prime}$ and discriminant $D$ over $R$. The space of CM Chow cycles of weight $2 k$ and level $N^{\prime}$ (for all discriminants $D$ ) over $R$, denoted by $C M_{k}\left(X\left(N^{\prime}\right)_{R}\right) \otimes \mathbb{R}$, is the $\mathbb{R}$-subspace generated by $s_{k}(\underline{x})$ for all CM points $\underline{x}$ in $X\left(N^{\prime}\right)(R)$.

Now we take $R$ to be an integral domain flat over $\mathbb{Z}$ and unramified over primes dividing $N$. Let $\underline{x} \in X_{0}(R)$ be a Heegner point of discriminant $D$ rational over $R$. We want to define the associated Heegner cycle $s_{k}(\underline{x})$ of weight $2 k$ over $X_{0}(N)_{R}$. The Kuga-Sato variety over $X_{0}(N)_{R}$ does not have a nice regular model. So we choose $N^{\prime}$ with $N \mid N^{\prime}$ to be any number which is a product of two coprime integers greater than or equal to 3 and such that $R$ is also unramified over primes dividing $N^{\prime}$. Let $\pi: X\left(N^{\prime}\right)_{R} \rightarrow X_{0}(N)_{R}$ be the natural projection, then $\pi^{*}(\underline{x})=u(\underline{x}) \sum_{i} \underline{x}_{i}$ for points $\underline{x}_{i}$ in $X\left(N^{\prime}\right)\left(R_{i}\right)$ with $\pi\left(\underline{x}_{i}\right)=\underline{x}$, where $u(\underline{x})=|\operatorname{Aut}(\underline{x})| / 2$ and $R_{i}$ is certain ring extension of $R$. Note that each elliptic curve $E_{i}$ represented by $\underline{x}_{i}$ has CM by $\mathcal{O}_{D}$ (independent of the choice of $i$ ). Applying the construction described above on each $x_{i}$ we obtain CM Chow cycles $s_{k}\left(\underline{x}_{i}\right) \in s_{k}\left(X\left(N^{\prime}\right)_{R_{i}}\right) \otimes \mathbb{R}$. Let

$$
s_{k}(\underline{x})=\frac{1}{u(\underline{x}) \sqrt{\operatorname{deg} \pi}} s_{k}\left(\pi^{*}(\underline{x})\right)=\frac{1}{\sqrt{\operatorname{deg} \pi}} \sum_{i} s_{k}\left(\underline{x}_{i}\right),
$$


then $s_{k}(\underline{x})$ is called a Heegner cycle of weight $2 k$ and level $\Gamma_{0}(N)$ over $R$. We also write $\operatorname{Heeg}_{k}\left(X_{0}(N)_{R}\right) \otimes \mathbb{R}$ for the space generated by all $s_{k}(\underline{x})$ with $\underline{x}$ a Heegner divisor rational over $R$ of $X_{0}(N)$. The construction of $\operatorname{Heeg}_{k}\left(X_{0}(N)_{R}\right) \otimes \mathbb{R}$ is Galois equivariant, and does not depend on the choice of the auxiliary number $N^{\prime}$.

The Hecke operators $T_{m}$ induce a natural action on $\operatorname{Heeg}_{k}\left(X_{0}(N)_{R}\right) \otimes \mathbb{R}$. If $T_{m}(\underline{x})=\sum_{i} \underline{x}_{i}$, then

$$
T_{m}\left(s_{k}(\underline{x})\right)=m^{k-1} \sum_{i} s_{k}\left(\underline{x}_{i}\right)
$$

and

$$
T_{m} T_{n}=\sum_{d \mid(m, n)} d^{k-1} T_{\frac{m n}{d^{2}}}
$$

Details of the above construction can be found in [9] over a field or in [11] in the general setting.

Now back to the situation of Section 1. We define

$$
s_{D, r}=\sum_{x \in P_{D, r}} s_{k}(x) \in \operatorname{Heeg}_{k}\left(X_{0}(N)_{K_{D}}\right) \otimes \mathbb{R}
$$

and

$$
s_{D, r}^{*}=\sum_{x \in P_{D, r}} s_{k}(x)+\sum_{x \in P_{D, r}} \overline{s_{k}(x)} \in \operatorname{Heeg}_{k}\left(X_{0}(N)_{\mathbb{Q}}\right) \otimes \mathbb{R},
$$

where the bar denotes the complex conjugate. Let $D$ be a fundamental discriminant, then $[8$, p. $542,(3)]$

$$
T_{m}\left(P_{D, r}\right)=\sum_{m=d d^{\prime}}\left(\frac{D}{d^{\prime}}\right) P_{D d^{2}, r d}
$$

which implies that

$$
T_{m} s_{D, r}^{*}=m^{k-1} \sum_{m=d d^{\prime}}\left(\frac{D}{d^{\prime}}\right) s_{D d^{2}, r d}^{*} .
$$

In particular, the space $\operatorname{Heeg}_{k}\left(X_{0}(N)_{\mathbb{Q}}\right) \otimes \mathbb{R}$ is stable under all Hecke operators $T_{m}$ for $(m, N)=1$.

Similarly we define an integral version

$$
\underline{s}_{D, r}=\sum_{x \in \underline{P}_{D, r}} s_{k}(\underline{x}) \in \operatorname{Heeg}_{k}\left(X_{0}(N)_{\mathcal{O}_{D}}\right) \otimes \mathbb{R}
$$

and

$$
\underline{s}_{D, r}^{*}=\sum_{\underline{x} \in \underline{P}_{D, r}} s_{k}(\underline{x})+\sum_{\underline{x} \in \underline{P}_{D, r}} \overline{s_{k}(\underline{x})} \in \operatorname{Heeg}_{k}\left(X_{0}(N)_{\mathbb{Z}}\right) \otimes \mathbb{R} .
$$

The construction of CM Chow cycles depends on the choice of the embedding $\tau: \operatorname{End}_{R}(E) \rightarrow \mathbb{C}$. If $\tau$ changes one has the following result [11, Prop. 2.4.1] or [9, p. $106]$.

Lemma 2.1. If $\tau$ changes to its complex conjugate then $s_{k}\left(E_{\bar{\tau}}\right)=(-1)^{k-1} s_{k}\left(E_{\tau}\right)$. 
In particular, the complex conjugate has the following effect on CM cycles

$$
\overline{s_{k}(x)}=(-1)^{k-1} s_{k}(\bar{x}) \in \operatorname{Heeg}_{k}\left(X_{0}(N)_{H_{D}}\right) \otimes \mathbb{R}
$$

Therefore

$$
\begin{aligned}
s_{D, r}^{*} & =\sum_{x \in P_{D, r}} s_{k}(x)+(-1)^{k-1} \sum_{x \in P_{D, r}} s_{k}(\bar{x}) \\
& =\sum_{x \in P_{D, r}} s_{k}(x)+(-1)^{k-1} \sum_{y \in P_{D,-r}} s_{k}(y) .
\end{aligned}
$$

On the other hand, if $f \in S_{2 k}\left(\Gamma_{0}(N)\right)$ is a normalized newform, then the $f$ isotypical component of $s_{D, r}$ has the following effect under the complex conjugate.

Lemma 2.2. The complex conjugate of the $f$-isotypical component $\left(s_{D, r}\right)_{f}$ of $s_{D, r}$ is given by

$$
\left(\overline{s_{D, r}}\right)_{f}=\left(-\varepsilon_{f}\right) \cdot\left(s_{D, r}\right)_{f},
$$

where $\varepsilon_{f}= \pm 1$ is the sign in the functional equation of $L(f, s)$.

Proof. See the proof of [9, Prop. 6.2].

By Lemma 2.2 if we assume further that $f \in S_{2 k}^{-}\left(\Gamma_{0}(N)\right)$, then $\varepsilon_{f}=-1$ and thus

$$
\left(s_{D, r}^{*}\right)_{f}=2\left(s_{D, r}\right)_{f} \in \operatorname{Heeg}_{k}\left(X_{0}(N)_{K_{D}}\right) \otimes \mathbb{R} \text {. }
$$

\section{Height pairing between Heegner cycles}

In this section, we always let $D_{1}, D_{2}<0$ denote coprime (but not necessarily fundamental) discriminants with $D_{i} \equiv r_{i}^{2}(\bmod 4 N)$, and let $\varrho$ be such that $\varrho \equiv$ $r_{1} r_{2}(\bmod 4 N)$. Our main goal is to compute the height pairing $\left\langle s_{D_{1}, r_{1}}^{*}, T_{m} s_{D_{2}, r_{2}}^{*}\right\rangle$ for $(m, N)=1$. The height pairing is defined in [11] through the arithmetic intersection theory developed by Gillet-Soulé [5]. Roughly speaking, let

$$
\widehat{s}_{D_{i}, r_{i}}^{*}=\left(\underline{s}_{D_{i}, r_{i}}^{*}, g_{D_{i}, r_{i}}\right)
$$

be the arithmetic cycle associated to $s_{D_{i}, r_{i}}^{*}$ with $g_{D_{i}, r_{i}}$ a Green current at infinity, then

$$
\left\langle s_{D_{1}, r_{1}}^{*}, T_{m} s_{D_{2}, r_{2}}^{*}\right\rangle=(-1)^{k}\left(\widehat{s}_{D_{1}, r_{1}}^{*} \cdot T_{m} \widehat{s}_{D_{2}, r_{2}}^{*}\right),
$$

where the product on the right hand side means the arithmetic intersection. This height pairing has the following decomposition into local height pairings and thus into the local intersections

$$
\begin{aligned}
\left\langle s_{D_{1}, r_{1}}^{*}, T_{m} s_{D_{2}, r_{2}}^{*}\right\rangle & =\sum_{p<\infty}\left\langle s_{D_{1}, r_{1}}^{*}, T_{m} s_{D_{2}, r_{2}}^{*}\right\rangle_{p}+\left\langle s_{D_{1}, r_{1}}^{*}, T_{m} s_{D_{2}, r_{2}}^{*}\right\rangle_{\infty} \\
& =(-1)^{k}\left(\sum_{p<\infty}\left(\underline{s}_{D_{1}, r_{1}}^{*} \cdot T_{m} \underline{s}_{D_{2}, r_{2}}^{*}\right)_{p} \log p\right)+\left\langle s_{D_{1}, r_{1}}^{*}, T_{m} s_{D_{2}, r_{2}}^{*}\right\rangle_{\infty} .
\end{aligned}
$$

We begin with the computation of the height pairing at non-archimedean places. Let $W$ be a complete local ring with a prime element $\pi$ and algebraically closed residue field of characteristic $p$ with $(p, N)=1$. Let $\underline{x}_{1} \in \underline{P}_{D_{1}, r_{1}}(W)$ and $\underline{x}_{2} \in \underline{P}_{D_{2}, \varepsilon r_{2}}(W)$ for $\varepsilon= \pm 1$. Suppose $\left(\underline{x}_{1} \cdot \underline{x}_{2}\right)_{W}>0$, and let $z=\left(E_{1} \rightarrow E_{1}^{\prime}\right)=\left(E_{2} \rightarrow E_{2}^{\prime}\right)$ be the common reduction of $\underline{x}_{1}$ and $\underline{x}_{2} \bmod (\pi)$. Let $B(p)=\operatorname{End}\left(E_{i}\right) \otimes \mathbb{Q}$, then $B(p)$ is 
the definite quaternion algebra ramified at $p$. Write $R=\operatorname{End}_{W / \pi W}(z)$, then $R$ is the right order of the left ideal class (which uniquely determines $z$ ) for the Eichler order of level $N$ in $B(p)$. We also define $S_{\left[D_{1}, 2 n, D_{2}\right]}$ to be the Clifford order associated to the quadratic form $\left[D_{1}, 2 n, D_{2}\right]$

$$
S_{\left[D_{1}, 2 n, D_{2}\right]}=\mathbb{Z}+\mathbb{Z} \frac{r_{1}+e_{1}}{2}+\mathbb{Z} \frac{r_{2}+e_{2}}{2}+\mathbb{Z} \frac{r_{1} r_{2}+r_{1} e_{2}+r_{2} e_{1}+e_{1} e_{2}}{4},
$$

with $e_{i}^{2}=D_{i}$ for $i=1,2$ and $e_{1} e_{2}+e_{2} e_{1}=2 n$. As pointed out in [8, pp. 549-551], the intersections over $W$ of $\underline{P}_{D_{1}, r_{1}}$ and $\underline{P}_{D_{2}, \varepsilon r_{2}}$ are in one-to-one correspondence with the embeddings of $S_{\left[D_{1}, 2 n, D_{2}\right]}$ into right orders $R$ of the distinct left ideal classes for the Eichler order of level $N$ in $B(p)$. More precisely, if $\phi: S_{\left[D_{1}, 2 n, D_{2}\right]} \rightarrow R$ is an embedding that corresponds to the intersection between $\underline{x}_{1} \in \underline{P}_{D_{1}, r_{1}}(W)$ and $\underline{x}_{2} \in \underline{P}_{D_{2}, \varepsilon r_{2}}(W)$ at $z$, then $\operatorname{End}(z) \cong R, \phi\left(e_{1}\right)=\sqrt{D_{1}}$ and $\phi\left(e_{2}\right)=\varepsilon \sqrt{D_{2}}$, where $\sqrt{D_{i}}$ means the image of the endomorphism $\sqrt{D_{i}} \in \operatorname{End}\left(\underline{x}_{i}\right)$ in $R$ under the reduction $\bmod \pi$.

Proposition 3.1. Suppose $\underline{x}_{i} \in \underline{P}_{D_{i}, r_{i}}(W)$ for $i=1,2$, and suppose they intersect at $z$ which corresponds to an embedding $\phi: S_{\left[D_{1}, 2 n, D_{2}\right]} \rightarrow R=\operatorname{End}(z)$, then

$$
\left(s_{k}\left(\underline{x}_{1}\right) \cdot \varepsilon^{k-1} s_{k}\left(\underline{x}_{2}\right)\right)_{W}=(-1)^{k-1} P_{k-1}\left(\frac{n}{\sqrt{D_{1} D_{2}}}\right) \cdot\left(\underline{x}_{1} \cdot \underline{x}_{2}\right)_{W} \cdot
$$

Proof. By $[11,(3.3 .1)]$ we have

$$
\left(s_{k}\left(\underline{x}_{1}\right) \cdot \varepsilon^{k-1} s_{k}\left(\underline{x}_{2}\right)\right)_{W}=\varepsilon^{k-1}\left(s_{k}\left(\underline{x}_{1}\right)_{0} \cdot s_{k}\left(\underline{x}_{2}\right)_{0}\right) \cdot\left(\underline{x}_{1} \cdot \underline{x}_{2}\right)_{W},
$$

where $s_{k}\left(\underline{x}_{1}\right)_{0}$ and $s_{k}\left(\underline{x}_{2}\right)_{0}$ denote reductions of $s_{k}\left(\underline{x}_{1}\right)$ and $s_{k}\left(\underline{x}_{2}\right) \bmod (\pi)$ respectively, and $\left(s_{k}\left(\underline{x}_{1}\right)_{0} \cdot s_{k}\left(\underline{x}_{2}\right)_{0}\right)$ denotes their intersection number inside the abelian variety $E_{1}^{2 k-2}=E_{2}^{2 k-2}$. To compute this intersection number we use the pairing on the $l$-adic cohomology (see [11, p. 129])

$$
H^{2 k-2}\left(E_{i}^{2 k-2}, \mathbb{Q}_{l}(k-1)\right) \times H^{2 k-2}\left(E_{i}^{2 k-2}, \mathbb{Q}_{l}(k-1)\right) \stackrel{(\cdot, \cdot)}{\rightarrow} \mathbb{Q}_{l},
$$

which is induced from the pairing on $H^{1}\left(E_{i}, \mathbb{Q}_{l}\right)$. Now we choose $l \neq p$ such that both $\sqrt{\left|D_{1}\right|}$ and $\sqrt{\left|D_{2}\right|}$ are in $\mathbb{Q}_{l}$, but $\sqrt{-1}$ is not in $\mathbb{Q}_{l}$ (such an $l$ always exists by the Chebotarev density theorem). Let $F=\mathbb{Q}_{l}(\sqrt{-1})$, after fixing an isomorphism $H^{1}\left(E_{1}, \mathbb{Q}_{l}\right) \otimes F \cong H^{1}\left(E_{2}, \mathbb{Q}_{l}\right) \otimes F$ we denote both spaces by $H$. We choose a basis $\{X, Y\}$ of $H$ such that

$$
\left[\phi\left(e_{1}\right)\right] X=-\sqrt{D_{1}} X, \quad\left[\phi\left(e_{1}\right)\right] Y=\sqrt{D_{1}} Y, \quad \text { and } \quad(X, Y)=\sqrt{-1} \in F .
$$

In other words, the action of $\left[\phi\left(e_{1}\right)\right]$ has the following matrix with respect to the basis $\{X, Y\}$

$$
\left[\phi\left(e_{1}\right)\right]=\left[\begin{array}{cc}
-\sqrt{D_{1}} & 0 \\
0 & \sqrt{D_{1}}
\end{array}\right]
$$

Similarly, let $\left\{X^{\prime}, Y^{\prime}\right\}$ be a basis of $H=H^{1}\left(E_{2}, \mathbb{Q}_{l}\right)$ such that

$$
\left[\phi\left(e_{2}\right)\right] X^{\prime}=-\varepsilon \sqrt{D_{2}} X^{\prime}, \quad\left[\phi\left(e_{2}\right)\right] Y^{\prime}=\varepsilon \sqrt{D_{2}} Y^{\prime}, \quad \text { and } \quad\left(X^{\prime}, Y^{\prime}\right)=\sqrt{-1} \in F
$$

Under the above setting we have the following result. 
Lemma 3.2. Suppose $\left[\begin{array}{l}X^{\prime} \\ Y^{\prime}\end{array}\right]=\left[\begin{array}{ll}a & b \\ c & d\end{array}\right]\left[\begin{array}{l}X \\ Y\end{array}\right]$, then

$$
s_{k}\left(\underline{x}_{1}\right)_{0} \cdot s_{k}\left(\underline{x}_{2}\right)_{0}=(-1)^{k-1} P_{k-1}(a d+b c),
$$

where $P_{k-1}(t)$ is the standard Legendre polynomial of degree $k-1$.

Proof. As $a d-b c=1$, this is [11, Prop. 3.3.3].

We next determine the matrix $\left[\begin{array}{ll}a & b \\ c & d\end{array}\right]$. Suppose the matrix of $\left[\phi\left(e_{2}\right)\right]$ with respect to the basis $\{X, Y\}$ is given by

$$
\left[\phi\left(e_{2}\right)\right]=\left[\begin{array}{ll}
\alpha & \beta \\
\gamma & \delta
\end{array}\right]
$$

Since

$$
\left[\phi\left(e_{1} e_{2}+e_{2} e_{1}\right)\right]=[2 n]
$$

we obtain

$$
\left[\begin{array}{cc}
-\alpha \sqrt{D_{1}} & 0 \\
0 & \delta \sqrt{D_{1}}
\end{array}\right]=\left[\begin{array}{cc}
n & 0 \\
0 & n
\end{array}\right]
$$

which implies that

$$
\alpha=\frac{-n}{\sqrt{D_{1}}}, \quad \delta=\frac{n}{\sqrt{D_{1}}}
$$

By (3.6)

$$
\left[\phi\left(e_{2}\right)\right] X^{\prime}=\left[\phi\left(e_{2}\right)\right](a X+b Y)=(a \alpha+b \gamma) X+(a \beta+b \delta) Y=-\varepsilon \sqrt{D_{2}} X^{\prime} .
$$

Thus

$$
a\left(\alpha+\varepsilon \sqrt{D_{2}}\right)+b \gamma=0
$$

and

$$
a=-\frac{\gamma}{\alpha+\varepsilon \sqrt{D_{2}}} b
$$

Similarly, by $\left[e_{2}\right] Y^{\prime}=\sqrt{D_{2}} Y^{\prime}$ we obtain

$$
c=-\frac{\gamma}{\alpha-\varepsilon \sqrt{D_{2}}} d
$$

Putting (3.9), (3.12) and (3.13) together,

$$
\begin{aligned}
a d+b c & =\frac{a d+b c}{a d-b c}=\frac{-\frac{\gamma}{\alpha+\varepsilon \sqrt{D_{2}}}-\frac{\gamma}{\alpha-\varepsilon \sqrt{D_{2}}}}{-\frac{\gamma}{\alpha+\varepsilon \sqrt{D_{2}}}+\frac{\gamma}{\alpha-\varepsilon \sqrt{D_{2}}}} \\
& =\frac{\frac{-2 n}{\sqrt{D_{1}}}}{-2 \varepsilon \sqrt{D_{2}}}=\frac{\varepsilon n}{\sqrt{D_{1} D_{2}}} .
\end{aligned}
$$

Now (3.1) follows from (3.2), (3.7), (3.14) and $P_{k-1}(\varepsilon t)=\varepsilon^{k-1} P_{k-1}(t)$. 
Proposition 3.3. Let $D_{i}=r_{i}^{2}-4 n_{i} N<0(i=0,1)$ be coprime fundamental discriminants and write $\Delta=D_{1} D_{2}, \varrho=r_{1} r_{2}$. Then the finite part of the height pairing between $s_{D_{1}, r_{1}}^{*}$ and $T_{m} s_{D_{2}, r_{2}}^{*}$ is given by

$$
\sum_{p<\infty}\left\langle s_{D_{1}, r_{1}}^{*}, T_{m} s_{D_{2}, r_{2}}^{*}\right\rangle_{p}=2 m^{k-1} \sum_{\substack{|n|<m \sqrt{\Delta} \\ n \equiv m \varrho(2 N)}} \sigma_{\chi}^{\prime}\left(\left(\frac{n+m \sqrt{\Delta}}{2}\right) \mathfrak{n}^{-1}\right) P_{k-1}\left(\frac{n}{m \sqrt{\Delta}}\right)
$$

where $\mathfrak{n}=\left(N, \frac{r_{1} r_{2}+\sqrt{\Delta}}{2}\right)$ is a primitive ideal of norm $N$ in $\mathbb{Q}(\sqrt{\Delta})$, and $\sigma_{\chi}^{\prime}(\mathfrak{a})$ for each ideal $\mathfrak{a}$ of $\mathbb{Q}(\sqrt{\Delta})$ is defined in (3.17).

Proof. For any pair of (not necessarily fundamental) discriminants $D_{1}$ and $D_{2}$, the local height pairing at a prime $p$ is given by

$$
\begin{aligned}
& \left\langle s_{D_{1}, r_{1}}^{*}, s_{D_{2}, r_{2}}^{*}\right\rangle_{p} \\
= & (-1)^{k}\left(s_{D_{1}, r_{1}}^{*} \cdot s_{D_{2}, r_{2}}^{*}\right)_{p} \log p \\
= & 2(-1)^{k}\left(\sum_{\underline{x} \in P_{D_{1}, r_{1}}} s_{k}(\underline{x})\right) \cdot\left(\sum_{\underline{y} \in P_{D_{2}, r_{2}}} s_{k}(\underline{y})+(-1)^{k-1} \sum_{\underline{y} \in P_{D_{2},-r_{2}}} s_{k}(\underline{y})\right) \log p \\
= & \frac{-1}{2^{t}} \sum_{\substack{n \equiv \varrho(2 N) \\
n^{2}<\Delta}} \sum_{R_{i}}\left|\left\{S_{\left[D_{1}, 2 n, D_{2}\right]} \rightarrow R_{i} \bmod R_{i}^{\times} / \pm 1\right\}\right| P_{k-1}\left(\frac{n}{\sqrt{\Delta}}\right) \frac{\operatorname{ord}_{p}(M)+1}{2} \log p \\
= & 2 \sum_{\substack{n \equiv \varrho(2 N) \\
n^{2}<\Delta}} P_{k-1}\left(\frac{n}{\sqrt{\Delta}}\right) \cdot \ell^{\prime}(M, 0)
\end{aligned}
$$

where $M=\frac{D_{1} D_{2}-4 n^{2}}{4 N}, \ell(M, s)=\sum_{d \mid M} \epsilon(d) d^{s}$, and we have used formula (3.1) together with the following two formulas from $[8, \mathrm{p} .551]$

$$
\begin{aligned}
& \left(P_{D_{1}, r_{1}} \cdot\left(P_{D_{1}, r_{1}}+P_{D_{2}, r_{2}}\right)\right)_{p} \\
= & \frac{1}{2^{t+1}} \sum_{\substack{n \equiv r_{1} r_{2}(2 N) \\
n^{2}<D_{1} D_{2}}} \sum_{R_{i}}\left|\left\{S_{\left[D_{1}, 2 n, D_{2}\right]} \rightarrow R_{i} \bmod R_{i}^{\times} / \pm 1\right\}\right| \frac{\operatorname{ord}_{p}(M)+1}{2}
\end{aligned}
$$

and

$$
\frac{-1}{2^{t+1}} \sum_{R_{i}}\left|\left\{S_{\left[D_{1}, 2 n, D_{2}\right]} \rightarrow R_{i} \bmod R_{i}^{\times} / \pm 1\right\}\right| \frac{\operatorname{ord}_{p}(M)+1}{2} \log p=\ell^{\prime}(M, 0) .
$$


By (2.6) we obtain

$$
\begin{aligned}
& \sum_{p}\left\langle s_{D_{1}, r_{1}}^{*}, T_{m} s_{D_{2}, r_{2}}^{*}\right\rangle_{p} \\
= & m^{k-1} \sum_{m_{i}=d_{i} d_{i}^{\prime}}\left(\frac{D_{1}}{d_{1}^{\prime}}\right)\left(\frac{D_{2}}{d_{2}^{\prime}}\right)\left(\sum _ { p } \left\langles_{\left.\left.D_{1} d_{1}^{2}, r_{1} d_{1}, s_{D_{2} d_{2}^{2}, r_{2} d_{2}}^{*}\right\rangle_{p}\right)}^{*} \sum_{=} \sum_{m_{i}=d_{i} d_{i}^{\prime}}\left(\frac{D_{1}}{d_{1}^{\prime}}\right)\left(\frac{D_{2}}{d_{2}^{\prime}}\right)\left(\sum_{\substack{x-1 d_{1} d_{2}(2 N) \\
|x|<d_{1} d_{2} \sqrt{\Delta}}} P_{k-1}\left(\frac{x}{d_{1} d_{2} \sqrt{\Delta}}\right) \ell^{\prime}\left(\frac{\Delta d_{1}^{2} d_{2}^{2}-x^{2}}{4 N}, 0\right)\right)\right.\right. \\
= & 2 m^{k-1} \sum_{\substack{n \equiv m_{\varrho}(2 N) \\
|n|<\sqrt{\Delta} m}} P_{k-1}\left(\frac{n}{m \sqrt{\Delta}}\right) \sum_{\substack{d_{i}^{\prime} \mid\left(n, m_{i}\right) \\
d_{1}^{\prime}}}\left(\frac{D_{2}}{d_{2}^{\prime}}\right) \ell^{\prime}\left(\frac{\Delta m^{2}-n^{2}}{4 N\left(d_{1}^{\prime} d_{2}^{\prime}\right)^{2}}, 0\right),
\end{aligned}
$$

where in the last line we set $n=d_{1}^{\prime} d_{2}^{\prime} x$ and interchanged the order of summation. By $[8$, p. $553,(3)]$ the inner sum is given by

$$
\sum_{d_{i}^{\prime} \mid\left(n, m_{i}\right)}\left(\frac{D_{1}}{d_{1}^{\prime}}\right)\left(\frac{D_{2}}{d_{2}^{\prime}}\right) \ell^{\prime}\left(\frac{\Delta m^{2}-n^{2}}{4 N\left(d_{1}^{\prime} d_{2}^{\prime}\right)^{2}}, 0\right)=\sigma_{\chi}^{\prime}\left(\left(\frac{n+m \sqrt{\Delta}}{2}\right) \mathfrak{n}^{-1}\right) .
$$

Now, (3.15) and (3.16) complete the proof.

We next consider the local height pairing at the archimedean place. Let

$$
Q_{k-1}(t)=\int_{0}^{\infty}\left(t+\sqrt{t^{2}-1} \cosh u\right)^{-k} d u
$$

and

$$
G_{k}\left(z, z^{\prime}\right)=\sum_{\gamma \in \Gamma_{0}(N)}-2 Q_{k-1}\left(1+\frac{\left|z-\gamma z^{\prime}\right|^{2}}{2 y(z) y\left(\gamma z^{\prime}\right)}\right)
$$

where $y(z)$ denotes the imaginary part of the complex variable $z$.

Lemma 3.4. For any two coprime discriminants $D_{1}$ and $D_{2}$, we have

$$
\left\langle s_{D_{1}, r_{1}}^{*}, s_{D_{2}, r_{2}}^{*}\right\rangle_{\infty}=G_{k}\left(P_{D_{1}, r_{1}}, P_{D_{2}, r_{2}}\right)+(-1)^{k-1} G_{k}\left(P_{D_{1}, r_{1}}, P_{D_{2},-r_{2}}\right),
$$

where $G_{k}\left(P_{1}, P_{2}\right)$ is defined by extending $G_{k}(x, y)$ bi-linearly to divisors $P_{1}$ and $P_{2}$.

Proof. By [11, Prop. 4.1.2] for $x \in P_{D_{1}, r_{1}}(\mathbb{C})$ and $y \in P_{D_{2}, \epsilon r_{2}}(\mathbb{C})$ one has

$$
\left\langle s_{k}(x), s_{k}(y)\right\rangle_{\infty}=\frac{1}{2} G_{k}(x, y) .
$$


Thus

$$
\begin{aligned}
& \left\langle s_{D_{1}, r_{1}}^{*}, s_{D_{2}, r_{2}}^{*}\right\rangle_{\infty} \\
= & 2\left\langle\sum_{x \in P_{D_{1}, r_{1}}} s_{k}(x), \sum_{y \in P_{D_{2}, r_{2}}} s_{k}(y)+(-1)^{k-1} \sum_{z \in P_{D_{2},-r_{2}}} s_{k}(z)\right\rangle_{\infty} \\
= & G_{k}\left(P_{D_{1}, r_{1}}, P_{D_{2}, r_{2}}\right)+(-1)^{k-1} G_{k}\left(P_{D_{1}, r_{1}}, P_{D_{2},-r_{2}}\right)
\end{aligned}
$$

as desired.

Proposition 3.5. The local height pairing $\left\langle s_{D_{1}, r_{1}}^{*}, T_{m} s_{D_{2}, r_{2}}^{*}\right\rangle_{\infty}$ is given by

$$
m^{k-1}\left(G_{k}\left(P_{D_{1}, r_{1}}, T_{m} P_{D_{2}, r_{2}}\right)+(-1)^{k-1} G_{k}\left(P_{D_{1}, r_{1}}, T_{m} P_{D_{2},-r_{2}}\right)\right) .
$$

Proof. Evident from Lemma 3.4.

Let $F(z)=\sum_{m=1}^{\infty} a_{m} e^{2 \pi i m z} \in S_{2 k}^{-}\left(\Gamma_{0}(N)\right)$ be such that its $m$-th Fourier coefficients are given by

$$
\begin{aligned}
a_{m}= & (m \sqrt{\Delta})^{k-1} \sum_{\substack{|n|<m \sqrt{\Delta} \\
n \equiv m \varrho(2 N)}} \sigma_{\chi}^{\prime}\left(\left(\frac{n+m \sqrt{\Delta}}{2}\right) \mathfrak{n}^{-1}\right) P_{k-1}\left(\frac{n}{m \sqrt{\Delta}}\right) \\
& -(m \sqrt{\Delta})^{k-1} \sum_{\substack{n>m \sqrt{\Delta} \\
n \equiv m \varrho(2 N)}} \sigma_{0, \chi}\left(\left(\frac{n+m \sqrt{\Delta}}{2}\right) \mathfrak{n}^{-1}\right) Q_{k-1}\left(\frac{n}{m \sqrt{\Delta}}\right) \\
& -(m \sqrt{\Delta})^{k-1} \sum_{\substack{n>m \sqrt{\Delta} \\
n \equiv-m \varrho(2 N)}} \sigma_{0, \chi}\left(\left(\frac{n+m \sqrt{\Delta}}{2}\right) \mathfrak{n}^{\prime-1}\right) Q_{k-1}\left(\frac{n}{m \sqrt{\Delta}}\right)
\end{aligned}
$$

where $\chi$ is the genus character associated the decomposition of $\Delta=D_{1} D_{2}, \mathfrak{n}=$ $\left(N, \frac{\varrho+\sqrt{\Delta}}{2}\right)$, and $\sigma_{0, \chi}(\mathfrak{a})$ and $\sigma_{\chi}^{\prime}(\mathfrak{a})$ denote the value and derivative of

$$
\sigma_{s, \chi}(\mathfrak{a})=\sum_{\mathfrak{b} \mid \mathfrak{a}} \chi(\mathfrak{b}) N(\mathfrak{b})^{s}
$$

at $s=0$ respectively. See [8, p. 530] or Section 4 for more detail of $F$.

Theorem 1. For $m \geq 1$ relatively prime to $N$, the Fourier coefficient $a_{m}$ of $F$ is given by

$$
a_{m}=\frac{\sqrt{\Delta}^{k-1}}{2}\left\langle s_{D_{1}, r_{1}}^{*}, T_{m} s_{D_{2}, r_{2}}^{*}\right\rangle .
$$


Proof. By [8, p. 556, Th. 2] (the factor $(m \sqrt{\Delta})^{k-1}$ is missed on the second line there)

$$
\begin{aligned}
a_{m}= & \sqrt{\Delta}^{k-1} \sum_{\substack{|n|<m \sqrt{\Delta} \\
n \equiv m \varrho(2 N)}} m^{k-1} P_{k-1}\left(\frac{n}{m \sqrt{\Delta}}\right) \sigma_{\chi}^{\prime}\left(\left(\frac{n+m \sqrt{\Delta}}{2}\right) \mathfrak{n}^{-1}\right) \\
& +\sqrt{\Delta}^{k-1} m^{k-1}\left(G_{k}\left(P_{D_{1}, r_{1}}, T_{m} P_{D_{2}, r_{2}}\right)+(-1)^{k-1} G_{k}\left(P_{D_{1}, r_{1}}, T_{m} P_{D_{2},-r_{2}}\right)\right) / 2 \\
= & \frac{\sqrt{\Delta}^{k-1}}{2}\left(\sum_{p<\infty}\left\langle s_{D_{1}, r_{1}}^{*}, T_{m} s_{D_{2}, r_{2}}^{*}\right\rangle_{p}\right)+\frac{\sqrt{\Delta}^{k-1}}{2}\left(\left\langle s_{D_{1}, r_{1}}^{*}, T_{m} s_{D_{2}, r_{2}}^{*}\right\rangle_{\infty}\right) \\
= & \frac{\sqrt{\Delta}^{k-1}}{2}\left\langle s_{D_{1}, r_{1}}^{*}, T_{m} s_{D_{2}, r_{2}}^{*},\right\rangle
\end{aligned}
$$

where in the second equation we have used Proposition 3.5.

\section{Consequences}

We retain the notation of Section 3, and let $D_{i}=r_{i}^{2}-4 n_{i} N<0(i=1,2)$ be two coprime fundamental discriminants with $(D, 2 N)=1$. In this section we also make the following assumption.

Assumption 4.1. The height pairing is positive definite on $\mathrm{Heeg}_{k}\left(X_{0}(N)_{\mathbb{Q}}\right) \otimes \mathbb{R}$.

This positive definiteness is a special case of the general conjectures of Gillet-Soulé [6] and Bloch-Beilinson [1] [2] on positive definiteness of the height pairing, also see Conjectures 1.1.1 and 1.3.1 in [11].

By Assumption 4.1 and Theorem 1, we have the following spectral decomposition of $s_{D_{i}, r_{i}}^{*}$ (see [11, Prop. 5.1.2] for the precise argument)

$$
s_{D_{i}, r_{i}}^{*}=\sum_{j}\left(s_{D_{i}, r_{i}}^{*}\right)_{f_{j}},
$$

where $\left\{f_{j}\right\}$ is an orthogonal eigenbasis for $S_{2 k}\left(\Gamma_{0}(N)\right)$ with $f_{1}=f$ and $\left(s_{D_{i}, r_{i}}^{*}\right)_{f_{j}}$ is the $f_{j}$-isotypical component of $s_{D_{i}, r_{i}}^{*}$, that is for for $m$ with $(m, N)=1$

$$
T_{m}\left(\left(s_{D_{i}, r_{i}}^{*}\right)_{f_{j}}\right)=a_{m}\left(f_{j}\right)\left(s_{D_{i}, r_{i}}^{*}\right)_{f_{j}} .
$$

Moreover, the $f_{j}$ can be chosen to have real Fourier coefficients.

Now define

$$
G(z)=\sum_{j}\left\langle\left(s_{D_{1}, r_{1}}^{*}\right)_{f_{j}},\left(s_{D_{2}, r_{2}}^{*}\right) f_{j}\right\rangle f_{j} \in S_{2 k}\left(\Gamma_{0}(N)\right) .
$$

Lemma 4.2. Suppose $(m, N)=1$, then

$$
a_{m}=\frac{\sqrt{\Delta}^{k-1}}{2} a_{m}(G) .
$$


Proof. By the spectral decomposition of $s_{D_{i}, r_{i}}^{*}$ and $\left\langle\left(s_{D_{1}, r_{1}}^{*}\right)_{f_{j}},\left(s_{D_{2}, r_{2}}^{*}\right)_{j_{j^{\prime}}}\right\rangle=0$ for $j \neq j^{\prime}$ we get

$$
\begin{aligned}
a_{m}(G) & =\sum_{j}\left\langle\left(s_{D_{1}, r_{1}}^{*}\right)_{f_{j}}, a_{m}\left(f_{j}\right)\left(s_{D_{2}, r_{2}}^{*}\right)_{f_{j}}\right\rangle \\
& =\left\langle\sum_{j}\left\langle\left(s_{D_{1}, r_{1}}^{*}\right)_{f_{j}}, \sum_{j} a_{m}\left(f_{j}\right)\left(s_{D_{2}, r_{2}}^{*}\right)_{f_{j}}\right\rangle\right. \\
& =\left\langle\left(s_{D_{1}, r_{1}}^{*}\right), T_{m}\left(s_{D_{2}, r_{2}}^{*}\right)\right\rangle .
\end{aligned}
$$

The lemma now follows from Theorem 1.

Theorem 2. Let $f \in S_{2 k}^{-}\left(\Gamma_{0}(N)\right)$ be a normalized newform. Then the subspace of $H_{\text {Heeg }}\left(X_{0}(N)_{\mathbb{Q}}\right) \otimes \mathbb{R}$ generated by $\left(s_{D, r}^{*}\right)_{f}$ (for all fundamental discriminants $D$ with $(D, 2 N)=1)$ has dimension 1 if $L^{\prime}(f, k) \neq 0$ and 0 if $L^{\prime}(f, k)=0$. Moreover,

$$
|D|^{\frac{k-1}{2}}\left(s_{D, r}^{*}\right)_{f}=c\left(\frac{r^{2}-D}{4 N}, r\right) \cdot s_{f}^{*}
$$

where $s_{f}^{*} \in\left(\operatorname{Heeg}_{k}\left(X_{0}(N)_{\mathbb{Q}}\right) \otimes \mathbb{R}\right)_{f}$ is independent of $D$ and $r$, and such that

$$
\left\langle s_{f}^{*}, s_{f}^{*}\right\rangle=\frac{(2 k-2) ! N^{k-1}}{2^{2 k-1} \pi^{k}(k-1) !\left(\phi_{f}, \phi_{f}\right)} \cdot L^{\prime}(f, k) .
$$

Proof. First let $D_{i}=r_{i}^{2}-4 n_{i} N<0$ be two coprime fundamental discriminants. By Lemma 4.2 it follows that $F-\frac{1}{2} \sqrt{\Delta}^{k-1} G$ is an old form in $S_{2 k}\left(\Gamma_{0}(N)\right)$, so $(G, f)=$ $2 \sqrt{\Delta}^{1-k}(F, f)$. But

thus

$$
(G, f)=(f, f)\left\langle\left(s_{D_{1}, r_{1}}^{*}\right)_{f},\left(s_{D_{2}, r_{2}}^{*}\right)_{f}\right\rangle,
$$

$$
\left\langle\left(s_{D_{1}, r_{1}}^{*}\right)_{f},\left(s_{D_{2}, r_{2}}^{*}\right)_{f}\right\rangle=2 \sqrt{\Delta}^{1-k} \frac{(F, f)}{(f, f)} .
$$

The inner product $(F, f)$ is given by $[8$, p. 536]

$$
(F, f)=\frac{i^{k-1} \Gamma\left(k-\frac{1}{2}\right)}{2^{k+1} \pi^{k+1 / 2}} L^{\prime}(f, k) r_{k, N, \Delta, \varrho, D_{1}}(f),
$$

where $r_{k, N, \Delta, \varrho, D_{1}}(f)$ is the cycle integral whose precise definition is not important here and can be found in $[8$, p. 518]. Theorem A of [8] gives the following formula regarding the value of this cycle integral

$$
r_{k, N, \Delta, \varrho, D_{1}}(f)=\left(\frac{i}{2 N}\right)^{1-k} \frac{c\left(n_{1}, r_{1}\right) c\left(n_{2}, r_{2}\right)(f, f)}{\left(\phi_{f}, \phi_{f}\right)},
$$

where $c(n, r)$ are Fourier coefficients of the Jacobi form $\phi_{f}$ that corresponds to $f$ as in (1.1).

Combining (4.1), (4.2) and (4.3) we conclude

$$
\begin{aligned}
\left\langle\left(s_{D_{1}, r_{1}}^{*}\right)_{f},\left(s_{D_{2}, r_{2}}^{*}\right)_{f}\right\rangle & =\frac{\Gamma\left(k-\frac{1}{2}\right) N^{k-1}}{2 \pi^{k+\frac{1}{2}} \Delta^{\frac{k-1}{2}}} \cdot \frac{c\left(n_{1}, r_{1}\right) c\left(n_{2}, r_{2}\right)}{\left(\phi_{f}, \phi_{f}\right)} \cdot L^{\prime}(f, k) \\
& =\frac{(2 k-2) ! N^{k-1}}{2^{2 k-1} \pi^{k}(k-1) ! \Delta^{\frac{k-1}{2}}} \cdot \frac{c\left(n_{1}, r_{1}\right) c\left(n_{2}, r_{2}\right)}{\left(\phi_{f}, \phi_{f}\right)} \cdot L^{\prime}(f, k) .
\end{aligned}
$$


On the other hand, by [11, Cor. 0.3 .2$]$ and (2.10)

$$
\begin{aligned}
\left\langle\left(s_{D_{i}, r_{i}}^{*}\right)_{f},\left(s_{D_{i}, r_{i}}^{*}\right)_{f}\right\rangle & =\frac{1}{2}\left\langle\left(s_{D_{i}, r_{i}}^{*}\right)_{f},\left(s_{D_{i}, r_{i}}^{*}\right)_{f}\right\rangle_{K_{D_{i}}} \\
& =\frac{(2 k-2) ! \sqrt{\left|D_{i}\right|} L^{\prime}(f, k)}{2^{4 k-2} \pi^{2 k}(f, f)} L\left(f, D_{i}, k\right)
\end{aligned}
$$

and by $[8$, p. 527 , Cor. 1]

so

$$
\frac{c\left(n_{i}, r_{i}\right)^{2}}{\left(\phi_{f}, \phi_{f}\right)}=\frac{(k-1) !\left|D_{i}\right|^{k-1 / 2}}{2^{2 k-1} \pi^{k} N^{k-1}} \cdot \frac{L\left(f, D_{i}, k\right)}{(f, f)},
$$

$$
\left\langle\left(s_{D_{i}, r_{i}}^{*}\right)_{f},\left(s_{D_{i}, r_{i}}^{*}\right)_{f}\right\rangle=\frac{(2 k-2) ! N^{k-1}}{2^{2 k-1} \pi^{k}(k-1) !\left|D_{i}\right|^{k-1}} \cdot \frac{c\left(n_{i}, r_{i}\right)^{2}}{\left(\phi_{f}, \phi_{f}\right)} \cdot L^{\prime}(f, k) .
$$

Comparing (4.4) and (4.5) we see that

$$
c\left(n_{2}, r_{2}\right)\left|D_{1}\right|^{\frac{k-1}{2}}\left(s_{D_{1}, r_{1}}^{*}\right)_{f}-c\left(n_{1}, r_{1}\right)\left|D_{2}\right|^{\frac{k-1}{2}}\left(s_{D_{2}, r_{2}}^{*}\right)_{f}
$$

has height 0 , so Assumption 4.1 implies that

$$
c\left(n_{2}, r_{2}\right)\left|D_{1}\right|^{\frac{k-1}{2}}\left(s_{D_{1}, r_{1}}^{*}\right)_{f}=c\left(n_{1}, r_{1}\right)\left|D_{2}\right|^{\frac{k-1}{2}}\left(s_{D_{2}, r_{2}}^{*}\right)_{f} .
$$

Next suppose $D_{1}$ and $D_{2}$ are not necessarily coprime. By [10, Lem. 3.2] we can find a fundamental discriminant $D^{\prime}=r^{\prime 2}-4 n^{\prime} N$ coprime to $D_{1}, D_{2}$ and $2 N$ with $c\left(n^{\prime}, r^{\prime}\right) \neq 0$. Applying (4.6) to the pairs $\left(D_{i}, D^{\prime}\right)$ for $i=1,2$, we get

$$
\left|D_{1}\right|^{\frac{k-1}{2}}\left(s_{D_{1}, r_{1}}^{*}\right)_{f}=\frac{c\left(n_{1}, r_{1}\right)}{c\left(n^{\prime}, r^{\prime}\right)}\left|D^{\prime}\right|^{\frac{k-1}{2}}\left(s_{D^{\prime}, r^{\prime}}^{*}\right)_{f}
$$

and

$$
\left|D_{2}\right|^{\frac{k-1}{2}}\left(s_{D_{2}, r_{2}}^{*}\right)_{f}=\frac{c\left(n_{2}, r_{2}\right)}{c\left(n^{\prime}, r^{\prime}\right)}\left|D^{\prime}\right|^{\frac{k-1}{2}}\left(s_{D^{\prime}, r^{\prime}}^{*}\right)_{f} .
$$

The proof of the theorem is now complete by letting $s_{f}^{*}=\frac{{\sqrt{\left|D^{\prime}\right|}}^{k-1}}{c\left(n^{\prime}, r^{\prime}\right)}\left(s_{D^{\prime}, r^{\prime}}^{*}\right)_{f}$.

Note that the dimension 1 assertion for the cohomology classes of $\left(s_{D, r}^{*}\right)_{f}$ (under the $l$-adic Abel-Jacobi map) is an immediate consequence of the main theorem of Nekovár [9], while our result holds at the level of Chow class space. It would be interesting to obtain an unconditional proof of Theorem 2 following Borcherds' approach [3].

\section{Acknowledgements}

The author would like to thank Gross, Kohnen, Zagier and Zhang for their pioneering work. Without their work the current paper would not exist.

\section{References}

[1] A. A. Bẹlinson, Height pairing between algebraic cycles, in $K$-theory, arithmetic and geometry (Moscow, 1984-1986), Vol. 1289 of Lecture Notes in Math., 1-25, Springer, Berlin (1987).

[2] S. Bloch, Height pairings for algebraic cycles, in Proceedings of the Luminy conference on algebraic $K$-theory (Luminy, 1983), Vol. 34, 119-145 (1984).

[3] R. Borcherds, The Gross-Kohnen-Zagier theorem in higher dimensions, Duke Math. J. 97 (1999), no. 2, 219-233.

[4] P. Deligne, Formes modulaire et représentation l-adiques, in Séminaire Bourbaki 1968/1969 exp. 355., 139-172. Lecture Notes in Math., Vol. 179, Springer, Berlin (1971). 
[5] H. Gillet and C. Soulé, Arithmetic intersection theory, Inst. Hautes Études Sci. Publ. Math. (1990), no. 72, 93-174 (1991).

[6] - Arithmetic analogs of the standard conjectures, in Motives (Seattle, WA, 1991), Vol. 55 of Proc. Sympos. Pure Math., 129-140, Amer. Math. Soc., Providence, RI (1994).

[7] B. Gross, Heegner points on $X_{0}(N)$, in Modular forms (Durham, 1983), Ellis Horwood Ser. Math. Appl.: Statist. Oper. Res., 87-105, Horwood, Chichester (1984).

[8] B. Gross, W. Kohnen, and D. Zagier, Heegner points and derivatives of L-series. II, Math. Ann. 278 (1987), no. 1-4, 497-562.

[9] J. Nekovár, Kolyvagin's method for Chow groups of Kuga-Sato varieties, Invent. Math. 107 (1992), no. 1, 99-125.

[10] N. Skoruppa and D. Zagier, Jacobi forms and a certain space of modular forms, Invent. Math. 94 (1988), no. 1, 113-146.

[11] S. Zhang, Heights of Heegner cycles and derivatives of L-series, Invent. Math. 130 (1997), no. $1,99-152$

Department of Mathematical Sciences, Clemson University, Clemson, SC 29634, USA

E-mail address: huixue@clemson.edu 